\title{
Swelling characterization and drug delivery kinetics of polyacrylamide-co-itaconic acid/chitosan hydrogels
}

\author{
A. Martínez-Ruvalcaba1, J. C. Sánchez-Díaz', F. Becerra², L. E. Cruz-Barba1, \\ A. González-Álvarez ${ }^{*}$ \\ ${ }^{1}$ Chemical Engineering Department, University de Guadalajara, Guadalajara 44430, Mexico \\ ${ }^{2}$ Chemistry Department, University de Guadalajara, Guadalajara 44430, Mexico
}

Received 14 October 2008; accepted in revised form 6 December 2008

\begin{abstract}
Hybrid polymeric networks composed of polyacrylamide and chitosan were developed to determine their swelling and ascorbic acid delivery kinetics at various chitosan concentrations. The hybrid acrylamide/chitosan hydrogels were synthesized in aqueous itaconic acid solution $(1 \% \mathrm{w} / \mathrm{w})$. Young's modulus was also evaluated for the hydrogels, and the results were correlated with the swelling properties. Swelling experiments were carried out using three different $\mathrm{pH}$ solutions: acidic ( $\mathrm{pH} 4$ buffer solution), neutral (distilled water) and basic ( $\mathrm{pH} 10$ buffer solution). The results of the swelling study showed that the swelling properties of the network varied with the changes of the $\mathrm{pH}$ in the swelling solution, as well as concentration of chitosan. When chitosan concentration increased, the swelling capacity diminished, and therefore Young's modulus increased. The results indicated that the swelling process followed a second order kinetics. The ascorbic acid diffusion inside the hydrogel follows a Fickian mechanism. The ascorbic acid diffusion coefficients are reported as a function of chitosan concentration.
\end{abstract}

Keywords: polymer gels, chitosan, swelling kinetics, mechanical properties, drug delivery

\section{Introduction}

Hydrogels are three-dimensional polymeric networks that have a solid-like appearance, formed by two or more components, one of which is a liquid present in high quantity. The capability of hydrogels to swell in water is due to the hydrophilic groups present in the polymer chains, while its mechanical resistance is due in part to the physical or chemical network cross-linking [1-3]. Interpenetrating polymer networks are defined as a combination of two or more polymers forming a network, which are synthesized in juxtaposition. However, most interpenetrating polymer networks do not interpenetrate on a molecular scale. Studies of reversible volume changes in response to $\mathrm{pH}$, ionic

*Corresponding author, e-mail: agonzalezalvarez@gmail.com (C) BME-PT and GTE concentration, etc. have been carried out for several kinds of polymeric networks [3-5].

The swelling behavior of any polymer network depends upon the nature of the polymer, polymersolvent compatibility and degree of cross-linking. However, in the case of ionic networks, swelling kinetics depends upon mass transfer limitations, ion exchange and ionic interaction [6]. Swelling kinetics of hydrogels can be classified as diffusion-controlled (Fickian) or relaxation-controlled (nonFickian). When diffusion into the hydrogel occurs much faster than the relaxation of the polymer chains, the swelling kinetics is said to be diffusioncontrolled [7].

Hydrogels can swell to profitable rates when placed into an appropriate environment, which means a 
specific $\mathrm{pH}$, temperature, electric field, light, pressure or specific molecule [6-12]. Several researchers have studied the swelling of $\mathrm{pH}$-sensitive hydrogels and the influence of this parameter in chemical, biological and physiological systems [13]. Hydrogels exhibiting $\mathrm{pH}$-sensitive swelling behavior have been usually swollen from ionic networks that can contain acidic or basic pendant groups. When these groups are ionized, a swelling osmotic pressure inside the material is built up, and fixed charges are trapped in the gel. As a result of the electrostatic repulsion, the uptake of solvent in the network is increased $[14,15]$.

Polyacrylamide forms a hydrogel with an outstanding swelling capacity, which is not substantially affected by the $\mathrm{pH}$ and the presence of electrolytes. Polyacrylamide gels are obtained by free-radical cross-linking copolymerization of acrylamide and N,N'-methylenebis(acrylamide) monomers. The properties of polyacrylamide hydrogels strongly depend on the initial degree of dilution of the monomers and, if the amount of water present at polymerization increases, the network's structure would not be firm [16]. Due to their high water retention, polyacrylamide hydrogels are widely used as biomaterials, and have been extensively studied for biomedical applications, such as drug delivery systems [17-19]. Different polymeric networks with a polyacrylamide basis have been synthesized and used for various applications [20-22]. Krusic et al. prepared semi-interpenetrating polymeric networks based on polyacrylamide/ poly(itaconic acid) and demonstrated that poly(itaconic acid) interacts with the polyacrylamide gel mainly by hydrogen bonding, resulting in the formation of an interpolymer complex [21].

Chitosan is a copolymer of N-acetyl-glucosamine and $\mathrm{N}$-glucosamine units distributed randomly or in blocks throughout the biopolymer chain, depending on the processing method used to obtain the biopolymer. Several interesting properties of chitosan, such as gel and film forming ability, bioadhesion, biodegradability and biocompatibility have been reported. Chitosan has received a great deal of attention in the pharmaceutical field due to its promising properties $[23,24]$. Because of the properties of chitosan and polyacrylamide, several works have explored the feasibility of a polyacrylamide/chitosan blend. These studies have reported different aspects of the characterization, biocompatibility, and release properties of polyacrylamide/chitosan hydrogels [25-27].

Synthesis method, swelling kinetics, and Young's moduli for a polyacrylamide/chitosan hybrid polymeric network at various chitosan concentrations, and different swelling media are reported in this work. A study to determine the ascorbic acid delivery behavior was also carried out, and the diffusion coefficients of ascorbic acid in the hydrogels were obtained as a function of chitosan concentration.

\section{Experimental}

\subsection{Materials}

Acrylamide (AM) 99\% and chitosan (CS) 99\% from Aldrich Chemical; 2,2'-azobis(2-amidinopropane) dihydrochloride (V-50) from Wako Chemicals; itaconic acid 98.5\% from Acros; and ascorbic acid 99\% from Merck were used as received. N,N'-methylenebisacrylamide (NMBA) from Scientific Polymer was recrystallized from a methanol solution. Doubly distilled deionized water was used.

\subsection{Preparation of hydrogels}

The polyacrylamide/chitosan (PAM-CS) hydrogels were synthesized using five different mass ratios $\left(m_{A M} / m_{C S}: 100 / 0,95 / 5,90 / 10,85 / 15,80 / 20\right)$. First a chitosan powder was dispersed in a $1.0 \mathrm{wt} \%$ itaconic acid aqueous solution, in order to obtain a solution with a concentration of $1.0 \mathrm{wt} \%$ chitosan. Then an AM/NMBA aqueous solution was prepared $\left(m_{N M B A} / m_{A M}=0.02\right)$. Both solutions were then perfectly mixed and left to polymerize at $50^{\circ} \mathrm{C}$ for $24 \mathrm{hr}$ in the presence of $\mathrm{V}-50\left(m_{V-50} / m_{A M}=\right.$ 0.02 ). The total solids concentration in the system was $6 \%$ relative to water.

The hydrogels, obtained in the shape of rods, were cut into disks, which were then immersed in water and left for several days to wash out any residual monomer. After washing, the hydrogels were left to dry at room temperature. In order to produce materials with similar dimensions, the dried disks were carefully sanded until the thickness ranged from 1.5 to $2 \mathrm{~mm}$ with diameters between 10 and $12 \mathrm{~mm}$. 


\subsection{Swelling behavior}

In order to study the swelling behavior, the disk samples (approximately $0.05 \mathrm{~g}$ ) were immersed in three different swelling solutions: water, $\mathrm{pH} 4.0$ buffer solution, and $\mathrm{pH} 10.0$ buffer solution. The samples were placed in the swelling solution and the weight of the swollen samples was measured against time after the excess surface water was removed by gently tapping the surface with a dry piece of filter paper. The degree of swelling $(H)$ for each disk sample at time $t$ was calculated using Equation (1):

$$
H=\frac{w_{t}-w_{0}}{w_{0}}
$$

where $w_{t}$ and $w_{0}$ are the sample's weight at any given time, and in the dry state, respectively.

\subsection{Mechanical properties}

Compression tests were done on the hydrogel disks once they were swollen to equilibrium conditions at $20^{\circ} \mathrm{C}$. These tests were carried out in a thermomechanical analyzer (TMA-7 Perkin-Elmer). Hydrogels swollen to equilibrium conditions were cut to yield rectangles and were properly measured. Young's modulus was obtained from the slope of the initial linear zone (less than 5\% strain) of the stress-strain curve according to Equation (2) [28]:

$$
\tau=-E(\lambda-1)
$$

where $\tau$ is the applied stress, and $\lambda$ is the deformation given by the ratio of the deformed $(h)$ to the initial thickness $\left(h_{0}\right)$ of the hydrogel disks, $\lambda=$ $-h / h_{0}$.

\subsection{Drug delivery measurements}

Previously measured (weight and dimensions) xerogels were loaded with ascorbic acid by immersing them in a drug saturated aqueous solution (333 g/l) until equilibrium was reached. The loaded hydrogels were then dried at room temperature for a week and weighed to obtain the concentration of $\mathrm{V}-\mathrm{C}$ in the xerogels.

To study the drug delivery kinetics the loaded xerogels were immersed in $200 \mathrm{ml}$ of distilled water at $30^{\circ} \mathrm{C}$ which were continuously stirred. In order to follow the delivery kinetics, the conductivity of the solution $[\mu \mathrm{S} / \mathrm{cm}]$ was measured at different times using an Orion Star 4 conductometer. The swelling experiments were carried out keeping the disks immersed. Conductivity vs. ascorbic acid concentration calibration curves were obtained using standard solutions $(0-30 \mathrm{mg} / \mathrm{l})$ at $30^{\circ} \mathrm{C}$. The amount of drug released at any given selected time $\left(M_{t}\right)$ was obtained from the calibration curve. The maximum amount of drug available for release $\left(M_{\infty}\right)$ was determined by gravimetric measurements.

\section{Results and discussion}

\subsection{Swelling properties and Young's modulus}

Polymerization of the acrylamide-chitosan system using low concentrations of cross-linking agent (NMBA), and using itaconic acid aqueous solutions to dissolve the chitosan creates a hybrid polymeric network [2]. The swelling media and the amount of chitosan were changed in order to determine the influence of $\mathrm{pH}$ and chitosan concentration in the polymeric network. The hydrogel samples were immersed in swelling solutions with different $\mathrm{pH}$. The amount of solution that penetrated into the hydrogel until equilibrium was reached (equilibrium swelling, $H_{\infty}$ ) was determined during the swelling process. An example of swelling kinetics (water uptake versus time) for the hydrogels at different polyacrylamide/chitosan mass ratios is depicted in Figure 1. This figure corresponds to hydrogels swollen in a basic environment. The figure shows a decrease in the equilibrium swelling when the hydrogel's chitosan content is increased

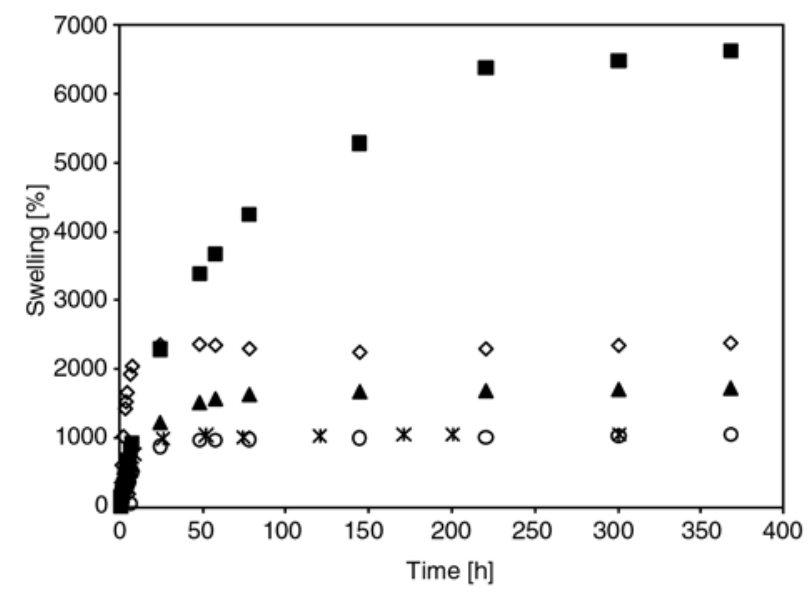

Figure 1. Swelling [\%] as function of time for hydrogels with different acrylamide/chitosan mass ratio $\left(m_{A M} / m_{C S}\right)(\boldsymbol{\square} 100 / 0, \diamond 95 / 5, \boldsymbol{\Delta} 90 / 10, \mathrm{O} 85 / 15$, $\star 80 / 20)$ 


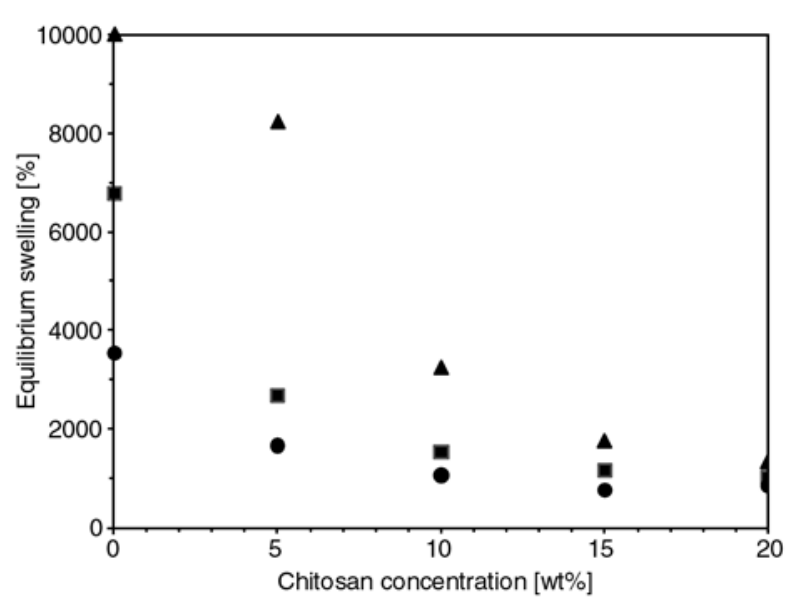

Figure 2. Equilibrium swelling [\%] as function of the chitosan concentration for the three different swelling media $(\bullet$ acid, $\boldsymbol{\Delta}$ water, $\boldsymbol{\square}$ basic)

(decrease of $m_{C S}$ ). This behavior suggests that the chitosan trapped in the hydrogel occupies more of the free space volume in the polymeric network, thus decreasing the volume available for swelling [2]. Besides, considering that the chitosan has a hydrophobic nature, when the chitosan concentration is increased, the hydrophilic character of the hydrogel is decreased. We can also observe how the mechanical properties (Young's modulus) of the hydrogel reach higher values when the chitosan concentration is increased [15] (Figures 2 and 3).

Figures 2 and 3 show the variation of $H_{\infty}$ and the Young's modulus, respectively, as a function of chitosan concentration. Addition of chitosan decreases the swelling capacity of the hydrogels and increases Young's modulus. This phenomenon may be explained due to the presence of ionizable groups in both, chitosan and itaconic acid. In acidic

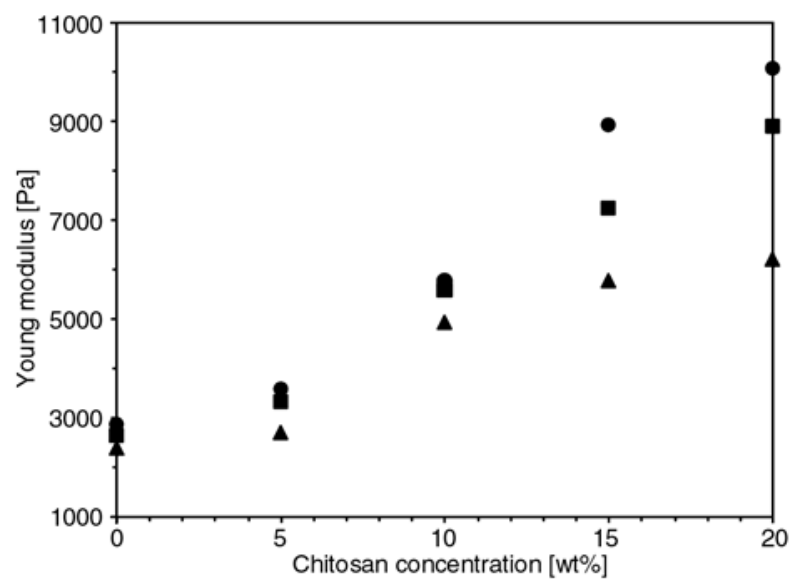

Figure 3. Young's modulus $[\mathrm{Pa}]$ as function of the chitosan concentration for the three different swelling media $(\bullet$ acid, $\boldsymbol{\Delta}$ water, $\boldsymbol{\square}$ basic) environments the amine groups in chitosan are ionized to form $\mathrm{NH}_{3}^{+}$groups, which allow chitosan to form networks through ionic links with the carboxy groups in the itaconic acid. When the chitosan concentration increases the cross-linking density increases, swelling capacity decreases and Young's modulus increases. In basic environments, the carboxy groups in the itaconic acid are ionized, while the chitosan $\mathrm{NH}_{3}^{+}$groups change back to $\mathrm{NH}_{2}$ groups. Under these conditions chitosan does not form ionic links and the cross-linking density decreases, the swelling capacity increases, and Young's modulus decreases. In a neutral $\mathrm{pH}$ environment (distilled water), the carboxy groups in the itaconic acid are ionized and the chitosan is not, therefore there is no ionic links, thus decreasing the cross-linking density. The ionic strength in the neutral swelling medium is smaller than that in acidic and basic media, therefore having the higher swelling capacities $[29,30]$. The hydrogels that are sensitive to the $\mathrm{pH}$ changes usually have ionizable groups; when these groups are ionized, for example in the chitosan amine groups at low $\mathrm{pH}$, is generated a swelling osmotic pressure inside the hydrogel. In addition, when ionized groups are deionized, the swelling osmotic pressure disappears, in the case of the amine groups of the chitosan it happens at high $\mathrm{pH}$ 's.

Figure 4 shows an example of the effect of the swelling medium's $\mathrm{pH}$. The figure corresponds to a hydrogel with an acrylamide/chitosan mass ratio of $90 / 10$. In water, $H_{\infty}$ is about $3500 \%$, while in a basic medium is just $1700 \%$, and in an acid medium is $1200 \%$. Considering that the swelling

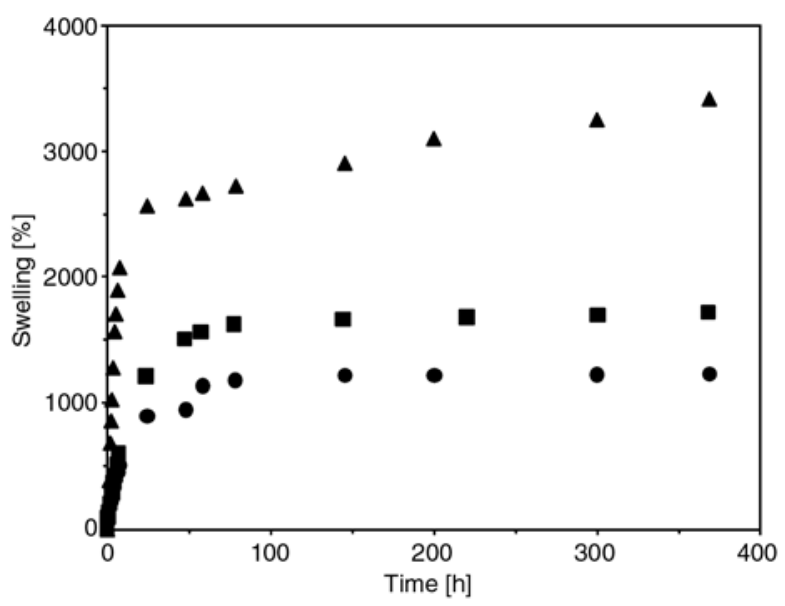

Figure 4. Effect of the swelling media over the swelling [\%] for the hydrogel with a mass ratio: $m_{A M} / m_{C S}=90 / 10(\bullet$ acid, $\boldsymbol{\Delta}$ water, $\boldsymbol{\square}$ basic $)$ 
process in the polymeric network is influenced by the swelling media's ionic strength, when the ionic strength increases, the concentration of ions in the hydrogel must increase in order to satisfy the Donnan equilibrium condition [15]; which establishes that the hydrogel swelling is diminished when the interactions of the material with the counter-ions of the swelling media increase.

\subsection{Swelling kinetics}

For most of the pharmaceutical applications it is important to know the swelling kinetics of the hydrogels, which will be used as drug release agents, because this process has a direct impact on drug release/delivery. There are a few rigorous theories dealing with hydrogel swelling kinetics; some authors have proposed that swelling can be described by a second order kinetics [30], as indicated in Equation (3):

$$
\frac{\mathrm{d} H}{\mathrm{~d} t}=k\left(H_{\infty}-H\right)^{2}
$$

where $k$ is the swelling rate constant.

By integration of Equation (3), and applying the initial conditions, we have Equations (4) and (5):

$$
\begin{aligned}
& \frac{t}{H}=\frac{1}{k_{\infty}}+\frac{t}{H_{\infty}} \\
& k_{\infty}=k H_{\infty}^{2}
\end{aligned}
$$

where $k_{\infty}$ is the equilibrium swelling rate constant. When the swelling kinetics corresponds to a second order kinetics, Equation (4) is a linear relationship, and $H_{\infty}$ and $k$ corresponds to the slope and intercept of the line, respectively [31]. The results obtained in this study agree with this behavior. Table 1 summarizes the second order swelling rate constants $(k)$ obtained.
Considering that the swelling process is affected by specific relations between the molecules of the swelling medium and the polymer pendant groups (amines, amides, carboxy), one can expect many kinds of polymer-solution interactions, and probably a complex kinetics. The decrease in the swelling rate when the chitosan concentration increases ( $k$ increases) suggests that specific interactions between the polymeric network loaded with chitosan and the medium are weaker when compared to the interactions that occur with the polymeric network without chitosan.

\subsection{Ascorbic acid delivery}

Results obtained from the tests of ascorbic acid release from the PAM/CS hydrogels in aqueous medium are shown in Figure 5. This figure shows the dependence of the release on the acrylamide/ chitosan mass ratio used. In addition to the PAM/CS hydrogels, results for a chitosan free sample (polyacrylamide hydrogel) are also shown. The figure shows that at constant time the drug released is lower when the chitosan concentration is higher. Taking into account that the swelling kinetics is

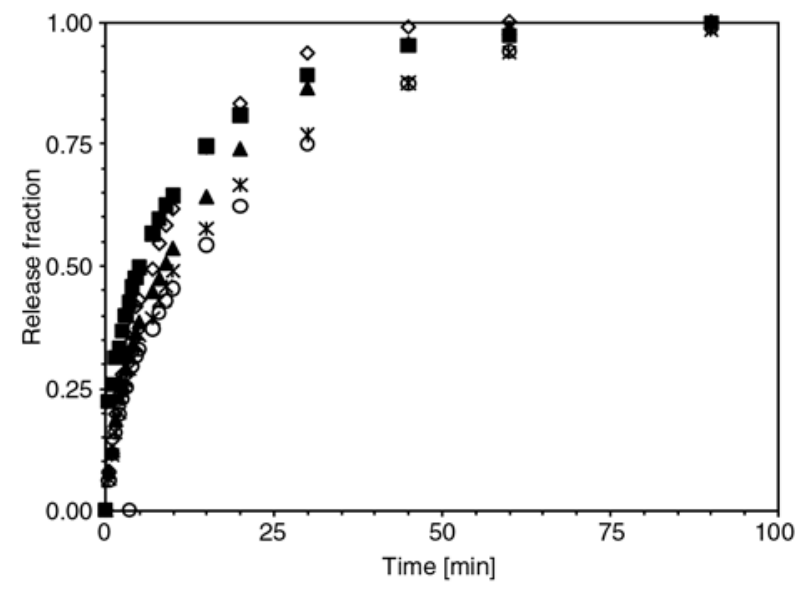

Figure 5. Release fraction versus time for hydrogels with different acrylamide/chitosan mass ratio $\left(m_{A M} / m_{C S}\right)$ immersed in a basic medium

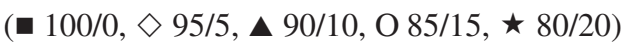

Table 1. Values of the swelling rate constant $k$ (g xerogel/g water-hr) for the PAM-CS hydrogels swelled in different aqueous media

\begin{tabular}{|c|c|c|c|}
\hline $\begin{array}{c}\text { Chitosanconcentration } \\
{[\mathbf{w t} \text { ) }]}\end{array}$ & $\begin{array}{c}\mathbf{k} \\
\text { (acid medium) }\end{array}$ & $\begin{array}{c}\mathbf{k} \\
\text { (water) }\end{array}$ & $\begin{array}{c}\mathbf{k} \\
\text { (basic medium) }\end{array}$ \\
\hline 0 & 0.028 & 0.025 & 0.020 \\
\hline 5 & 0.038 & 0.046 & 0.050 \\
\hline 10 & 0.085 & 0.077 & 0.088 \\
\hline 15 & 0.123 & 0.097 & 0.134 \\
\hline 20 & 0.289 & 0.610 & 0.340 \\
\hline
\end{tabular}


determined by the hydrogel porosity (volume of the free spaces in the polymeric network) [2], when the chitosan chains are trapped into the polymeric network, the chitosan occupies a fraction of that free space in the network. This causes a decrease in the hydrogel porosity, which is evidenced by the fact that the quantity of drug released decreases when chitosan concentration is increased. Considering that the ascorbic acid delivery is also governed by specific dipole-dipole interactions and hydrogen bonds between water molecules and the ions present in the solution, the decrease in the drug released when the chitosan concentration is increased is also influenced by the decrease in the chitosan degree of deionization, which improves hydrogel link formation, and therefore increases cross-linking density. That is the same phenomena observed for Young's modulus, which are higher when the chitosan concentration is increased.

Diffusion from the gel phase to the surroundings can be considered as a one-dimensional isothermic process for hydrogel samples with a small thickness respect to its diameter (the relationship between diameter and thickness must range between 5 and 8) [32]. In order to produce materials with appropriate dimensions, the sample diameters varied between 10 and $12 \mathrm{~mm}$, and thickness ranged from 1.5 to $2 \mathrm{~mm}$.

For a process controlled by diffusion in thin disks, Equation (6) is used to determine the nature of diffusion [33]:

$F=\frac{M_{t}}{M_{\infty}}=k_{1} t^{n}$

where the release fraction $(F)$ is defined as $M_{t} / M_{\infty}$, $M_{t}$ is the amount of drug released at any given time, $M_{\infty}$ is the maximum amount (weight) available for release, $t$ is the release time, $k_{1}$ is the kinetic constant of the system, and $n$ is a characteristic power for the drug release.

Equation (6) describes the release kinetics of a drug and is only valid for the first $60 \%$ of the fractional uptake. When $n$ is equal to 0.5 the drug is said to diffuse with Fickian behavior. For $n=1$ the behavior is called Case II diffusion. Finally, anomalous transport behavior, which is intermediate between Fickian and Case II, is known as non-Fickian diffusion [34]. For Fickian behavior, $k_{1}$ is given by Equation (7):
Table 2. Parameters $k_{1}, n$ and diffusion coefficients for the PAM-CS hydrogels at different chitosan concentrations

\begin{tabular}{|c|c|c|c|}
\hline $\begin{array}{c}\text { Chitosan concentration } \\
{[\mathbf{w t} \%]}\end{array}$ & $\mathbf{k}_{\mathbf{1}}$ & $\mathbf{n}$ & $\begin{array}{c}\mathbf{D} \\
{\left[\mathbf{c m}^{\left.\mathbf{2} \cdot \mathbf{s}^{-1}\right]}\right.}\end{array}$ \\
\hline 0 & 0.0296 & 0.495 & $1.48 \cdot 10^{-5}$ \\
\hline 5 & 0.0235 & 0.507 & $1.15 \cdot 10^{-5}$ \\
\hline 10 & 0.0198 & 0.514 & $8.33 \cdot 10^{-6}$ \\
\hline 15 & 0.0191 & 0.506 & $7.74 \cdot 10^{-6}$ \\
\hline 20 & 0.0172 & 0.512 & $3.19 \cdot 10^{-6}$ \\
\hline
\end{tabular}

$k_{1}=4\left(\frac{D_{i}}{\pi h^{2}}\right)^{\frac{1}{2}}$

where $D_{i}$ is the diffusion coefficient for the drug delivery mechanism and $h$ is the thickness of the loaded sample.

The power $n$ in Equation (6) was determined from slope of the release fraction $(F)$ vs. time log-log plot. The values of $\mathrm{n}$ shown in Table 2 indicate that the ascorbic acid release from the hydrogel follows a Fickian diffusion for all chitosan concentrations. This behavior suggests that the release process is controlled by diffusion only [2]. The values of $n$ are used together with Equation (6) in order to calculate the release kinetic constant $\left(k_{1}\right)$, which are also reported in Table 2 for different chitosan concentrations. The kinetic constants are lower when the chitosan concentration increases, and this fact suggests that the diffusion rate decreases when chitosan quantity increases due to the decrease in size of the free spaces in the network.

The diffusion coefficient $\left(D_{i}\right)$ for the ascorbic acid was determined using Equation (7), due to the fact that kinetic constants suggest Fickian behavior. Table 2 shows the $D_{i}$ values for different chitosan concentrations.

The diffusion coefficients obtained are inversely proportional to the chitosan concentration, and therefore the release rate decreases when the chitosan amount increases. This behavior confirms that an increase in the hydrogel's chitosan concentration reduces the swelling in neutral medium, and release rate and Young's modulus increase.

\section{Conclusions}

The synthesis of a hybrid polymeric network based in polyacrylamide and chitosan in acidic solution (itaconic acid) was carried out in order to determine 
its swelling kinetics and ascorbic acid release. Swelling capacity, equilibrium swelling and Young's modulus reached at different $\mathrm{pH}$ environments, as well as release fractions and diffusion coefficients for the ascorbic acid release were reported. It was found that the swelling capacity decreases and the Young's modulus increases when the chitosan concentration is increased, most probably due to the changes in the hydrogel porosity, which in turn causes an increase in the cross-linking density, as well as the hydrophobic character of chitosan, which reduces the hydrophilicity of the hydrogel.

The swelling kinetics and mechanical properties were studied using three different swelling media: acidic, neutral, and basic. In acidic medium the swelling capacity decreases due to the chitosan $\mathrm{NH}_{3}^{+}$groups that is ionically linked with the itaconic acid $\mathrm{COO}^{-}$group, and this ionic bond increases the cross-linking density. In aqueous medium, the $\mathrm{NH}_{3}^{+}$group loses a proton and becomes $\mathrm{NH}_{2}$, thus reducing the cross-linking density. In basic medium the swelling capacity decreases relative to the aqueous medium, because the itaconic acid is ionized and is forming hydrogen bonds with the nitrogen of the amine groups. The swelling of the hydrogels follows a second order kinetics, and the swelling rate constants obtained confirm the presence of different polymer-solution interactions in the system, which do not exist in the chitosan-free hydrogel.

The ascorbic acid release follows Fick's second law of diffusion, and the characteristic power, $n$, is about 0.5 . The kinetic constant $\left(k_{1}\right)$ and the diffusion coefficients decrease when the chitosan concentration in the hydrogel is increased, mainly due to a rise in the cross-linking density and a decrease on the volume of free spaces available in the hydrogel. The diffusion coefficients are between $10^{-5}$ and $10^{-6} \mathrm{~cm}^{2} / \mathrm{s}$, which suggest that the hydrogels studied have a good potential for their use in applications such as drug release, since similar values have been reported in papers with a pharmaceutical approach.

\section{References}

[1] Almdal K., Dyre J., Hvidt S., Kramer O.: What is a gel? Macromolecular Symposia, 76, 49-51 (1993).
[2] Berger J., Reist M., Mayer J. M., Felt O., Peppas N. A., Gurny R.: Structure and intereactions in covalently and ionically crosslinked chitosan hydrogels for biomedical applications. European Journal of Pharmaceutics and Biopharmaceutics, 57, 19-34 (2004). DOI: $10.1016 /$ S0939-6411(03)00161-9

[3] Sperling L. H.: Interpenetrating polymer networks and related materials. Plenum Press, New York (1981).

[4] Jeong S., Park S. J., Shin M-S., Kim S. I.: Characteristics of electrical responsive chitosan/polyallylamine interpenetrating polymer network hydrogel. Journal of Applied Polymer Science, 86, 2290-2295 (2002). DOI: 10.1002/app.11217

[5] Kim S. J., Shin S. R., Lee Y. M., Kim S. I.: Swelling characterizations of chitosan and polyacrylonitrile semi-interpenetrating polymer network hydrogels. Journal of Applied Polymer Science, 87, 2011-2015 (2003).

DOI: 10.1002/app.11699

[6] Frank S., Lauterbur P. C.: Voltage-sensitive magnetic gels as magnetic resonance monitoring agents. Nature, 363, 334-336 (1993). DOI: $10.1038 / 363334 \mathrm{a} 0$

[7] Peppas N. A., Colombo P.: Analysis of drug release behavior from swellable polymer carriers using the dimensionality index. Journal of Controlled Release, 45, 35-40 (1997). DOI: $10.1016 / \mathrm{S} 0168-3659(96) 01542-8$

[8] Tanaka T.: Phase transitions of gels. in 'Polyelectrolyte gels: Properties, preparation and applications' (eds.: Harland R. S., Prud'homme R. K.) American Chemical Society, Washington, Vol 480, 1-21 (1992).

[9] Suzuki A., Ishii T., Maruyama Y.: Optical switching in polymer gels. Journal of Applied Physics, 80, 131136 (1996). DOI: $10.1063 / 1.362768$

[10] Khare A. R., Peppas N. K.: Swelling/deswelling of anionic copolymer gels. Biomaterials, 16, 559-567 (1995).

DOI: $10.1016 / 0142-9612(95) 91130-Q$

[11] Zhong X., Wang Y-X., Wang S-C.: Pressure dependence of the volume phase-transitions of temperaturesensitive gels. Chemical Engineering Science, 51, 3235-3239 (1996). DOI: 10.1016/0009-2509(95)00344-4

[12] Peppas N. A., Huang Y.: Polymer and gels as molecular recognition agents. Pharmaceutical Research, 19, 578-587 (2002). DOI: 10.1023/A:1015389609344

[13] Peppas N. A., Khare A. R.: Preparation, structure and diffusional behavior of hydrogels in controlled release. Advanced Drug Delivery Reviews, 11, 1-35 (1993) DOI: $10.1016 / 0169-409 X(93) 90025-Y$

[14] Brannon-Peppas L., Peppas N. A.: Equilibrium swelling behavior of $\mathrm{pH}$-sensitive hydrogels. Chemical Engineering Science, 46, 715-722 (1991). DOI: $10.1016 / 0009-2509(91) 80177-Z$ 
[15] Peppas N. A., Bures P., Leobandung W., Ichikawa H.: Hydrogels in pharmaceutical formulations. European Journal of Pharmaceutics and Biopharmaceutics, 50, 27-46 (2000).

DOI: $10.1016 / \mathrm{S} 0939-6411(00) 00090-4$

[16] Naghash H. J., Okay O.: Formation and structure of polyacrylamide gels. Journal of Applied Polymer Science, 60, 971-979 (1996).

DOI: $10.1002 /($ SICI $) 1097-4628(19960516) 60: 7<971::$ AID-APP7>3.0.CO;2-J

[17] Blanco M. D., Garcia O., Olmo R., Teijon J. M., Katime I.: Release of 5-fluorouracil from poly(acrylamide-co-monopropyl itaconate) hydrogels. Journal of Chromatography B: Biomedical Sciences and Applications, 680, 243-253 (1996).

DOI: $10.1016 / 0378-4347(95) 00401-7$

[18] Chen J., Haesun P., Park K.: Synthesis of superporous hydrogels: Hydrogels with fast swelling and superabsorbent properties. Journal of Biomedical Materials Research Part A, 44, 53-62 (1999).

DOI: $\underline{10.1002 /(\mathrm{SICI}) 1097-4636(199901) 44: 1<53: \text { : }}$

$$
\text { AID-JBM6>3.0.CO;2-W }
$$

[19] Ferreira L., Vidal M. M., Gil M. H.: Design of a drugdelivery system based on polyacrylamide hydrogels. Evaluation of structural properties. The Chemical Educator, 6, 100-103 (2001).

DOI: $10.1333 / \mathrm{s} 00897010461 \mathrm{a}$

[20] Muniz E. C., Geuskens G.: Compressive elastic modulus of polyacrylamide hydrogels and semi-IPNs with poly(N-isopropylacrylamide). Macromolecules, 34, 4480-4484 (2001).

DOI: $10.1021 / \mathrm{ma} 0011921$

[21] Krusic K. M., Dzunuzovic E., Trifunovic S., Filipovic J.: Semi-IPNs based on polyacrylamide and poly(itaconic acid). Polymer Bulletin, 51, 159-166 (2003). DOI: $10.1007 / \mathrm{s} 00289-003-0203-7$

[22] Mishra S., Bajpai R., Katare R., Bajpai A. K.: Radiation induced crosslinking effect on semi-interpenetrating polymer networks of poly(vinyl alcohol). Express Polymer Letters, 1, 407-415 (2007).

DOI: $\underline{10.3144 / \text { expresspolymlett.2007.58 }}$

[23] Khor E., Lim L. Y.: Implantable applications of chitin and chitosan. Biomaterials, 24, 2339-2349 (2003). DOI: 10.1016/S0142-9612(03)00026-7

[24] Boonsongrit Y., Mitrevej A., Mueller B. W.: Chitosan drug binding by ionic interaction. European Journal of Pharmaceutics and Biopharmaceutics, 62, 267-274 (2006).

DOI: $10.1016 /$ j.ejpb.2005.09.002
[25] Risbud M. V., Bhonde R. R.: Polyacrylamide-chitosan hydrogels: In vitro biocompatibility and sustained antibiotic release studies. Drug Delivery, 7, 69-75 (2000).

DOI: $10.1080 / 107175400266623$

[26] Yazdani-Pedram M., Lagos A., Retuert P. J.: Study of the effect of reaction variables on grafting of polyacrylamide onto chitosan. Polymer Bulletin, 48, 93-98 (2002). DOI: $10.1007 / \mathrm{s} 00289-002-0006-2$

[27] Xia Y., Guo T., Song M., Zhang B., Zhang B.: Hemoglobin recognition by imprinting in semi-interpenetrating polymer network hydrogel based on polyacrylamide and chitosan. Biomacromolecules, 6, 26012606 (2005).

DOI: $\underline{10.1021 / \mathrm{bm} 0503241}$

[28] Ferry J. D.: Viscoelastic properties of polymers. Wiley, New York, (1980).

[29] Lárez C., Canelón F., Millán E., Katime I.: Interpolymeric complexes of poly(itaconic acid) and chitosan. Polymer Bulletin, 48, 361-366 (2002). DOI: $10.1007 / \mathrm{s} 00289-002-0049-4$

[30] Lee J. W., Kim S. Y., Kim S. S., Lee Y. M., Lee K. H., Kim S. J.: Synthesis and characteristics of interpenetrating polymer network hydrogel composed of chitosan and poly(acrylic acid). Journal of Applied Polymer Science, 73, 113-120 (1999).

DOI: 10.1002/(SICI)1097-4628(19990705)73:1<113:: AID-APP13>3.0.CO;2-D

[31] Katime I. A., Katime O., Katime D.: Smart materials of this millenium: Macromolecular hydrogels (in Spanish). Servicio Editorial de la Universidad del País Vasco, Bilbao (2004).

[32] Leanirith Y., Bunel C., Vairon J. P.: Reversible immobilization of drugs on a hydrogel matrix, 2. Diffusion of free chloramphenicol from poly(2-hydroxyethyl methacrylate) hydrogels. Die Makromolekulare Chemie, 191, 1119-1129 (1990). DOI: $10.1002 / \mathrm{macp} .1990 .021910514$

[33] Mathur A. M., Moorjani S. K., Scranton A. B.: Methods for synthesis of hydrogel networks: A review. Polymer Reviews, 36, 405-430 (1996). DOI: $\underline{10.1080 / 15321799608015226}$

[34] Karadag E., Saraydin D., Sahiner N., Guven O.: Radiation induced acrylamide/citric acid hydrogels and their swelling behaviors. Journal of Macromolecular Science Part A: Pure and Applied Chemistry, 38, 1105-1121 (2001). DOI: $10.1081 / \mathrm{MA}-100107132$ 